\title{
Energy Efficiency Analysis through Misalignment on New Design of Hexagonal Coil Array in Wireless Power Transfer
}

\author{
Taufik Hidayat $^{1^{*}}$, Sianturi Tigor Franky $D^{2}$, Rahutomo Mahardiko \\ ${ }^{1}$ Department of Computer Engineering, \\ Universitas Wiralodra, Indramayu, INDONESIA \\ ${ }^{2}$ Department of Electrical Engineering, \\ Universitas Mercu Buana, Jakarta, INDONESIA \\ ${ }^{3}$ Department Software Service, \\ Platinumetrix Pte. Ltd, Jakarta, INDONESIA
}

*Corresponding Author

DOI: https://doi.org/10.30880/ijie.2020.12.08.019

Received 7 November 2019; Accepted 6 May 2020; Available online 31 August 2020

\begin{abstract}
Nowadays, the transportation changes are happening in the Electric Vehicle (EV). Changes are made from cable to Wireless Power Transfer (WPT) as a solution. Current state of the implementation, there is still a misalignment on Power Transfer Efficiency (PTE) because of parking behavior. To overcome the problem, we propose new coil design for better optimization of the PTE. In addition, the new design has better size reduction of receiver (Rx) coil. Our experimental research is about to simulate Rx and transmitter (Tx) designs in simple and small scale of parking situations. The results show that an incremental PTE in the center of the coil, an incremental PTE during misalignment on a radius array, and an incremental PTE during misalignment tangential boundary. We conclude that overall better PTE is achieved compared to single coil design and circular coil arrays.
\end{abstract}

Keywords: Electric Vehicle (EV), Power Transfer Efficiency (PTE), hexagonal coil, misalignment, receiver $(\mathrm{Rx})$ and transmitter $(\mathrm{Tx})$ coil

\section{Introduction}

From our understanding about WPT, it is a very advanced technology to transmit energy with indirect connection between source and destination [1]. Lots of applications are implementing this technology, such as: (1) electric vehicles (EV), (2) capsule endoscopy, (3) power supplies (underwater or beneath the earth) and so on without any affection [1], [2], [3].

Power transfer applications demand has significantly increased especially in high power. Basically, the required power level must be handled well by converters to transmit power between primary and secondary sides [4], [5]. The problem is that the semiconductor device limitations limit the latest WPT system [6]. In addition, the low reliability of the WPT system is based on magnetic pairing with a single transmitter and receiver because it only has one energy transmission channel [7]. This is not suitable for applications that require power up to hundreds of KVA to MW. Therefore, WPT applications with various conditions including large incompatibilities must be able to meet the requirements with high efficiency transmission [8]. Lately, EV is one of the most common applications of WPT technology [9]. 
There are 2 types of WPT for EV, such as: (1) WPT static charging where EV needs steady position to charge the battery and (2) dynamic charging where EV can charge continuously the battery in moving condition as far as the railway grid [10]. Wireless charging make battery to have smaller capacity by cutting down up to $20 \%$, so that charging times will be short [11]. Fig. 1 shows the diagram where the Tx is placed on the ground and the Rx is mounted on the EV. Wireless charging is reached when EV is parked above Tx [12].

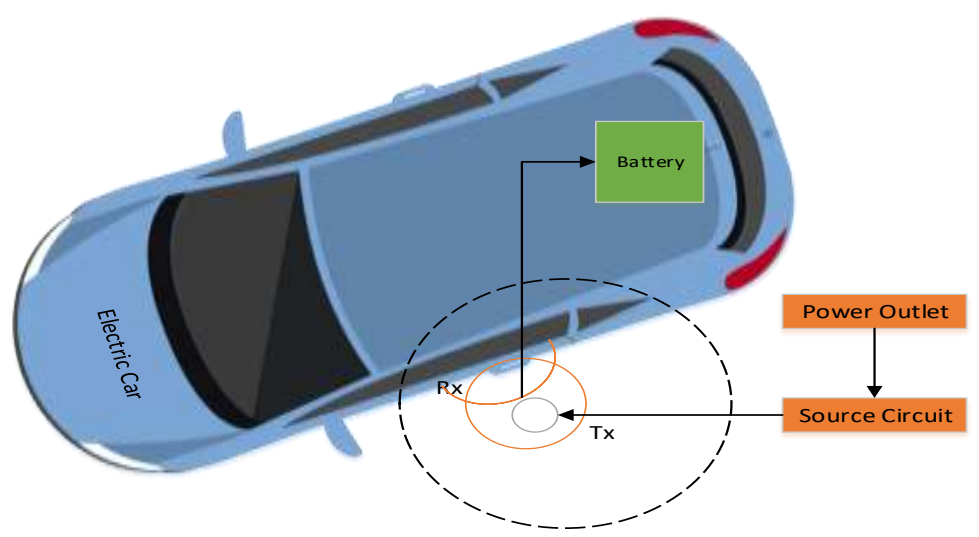

Fig. 1 - Charging EV diagram [13]

However, it may be practically difficult or impossible to park appropriately the EV so that Rx is completely aligned with Tx. Only 5\% drivers regarding the driver behavior can park in alignment tolerance. The result is that 5\% drivers have opportunity to achieve $95 \%$ of peak efficiency. If the condition is not met, less than $50 \%$ of peak efficiency will be obtained because of $15-20 \mathrm{~cm}$ for lateral misalignment [12].

Sometimes, if the distance between Tx and Rx is far away, the efficiency will be reduced significantly [14]. Because of the reason, many scientists are focusing on how to develop better PTE on EV. We explore that not only the distance but also the design and location of the coil structure affect PTE [12], [13]. The affection of PTE is called misalignment when the weak coupling is happening between Tx and Rx. The lateral and angular misalignment is highly happening in current WPT technology [15]. From new study about array circle coil design for Tx and circle coil for Rx, the combination cannot reduce the PTE, meanwhile it only reduces the load on EV. In addition, the decreased PTE also happens in tangential boundary area misalignment [16]. Fig. 2 shows array circular coil.

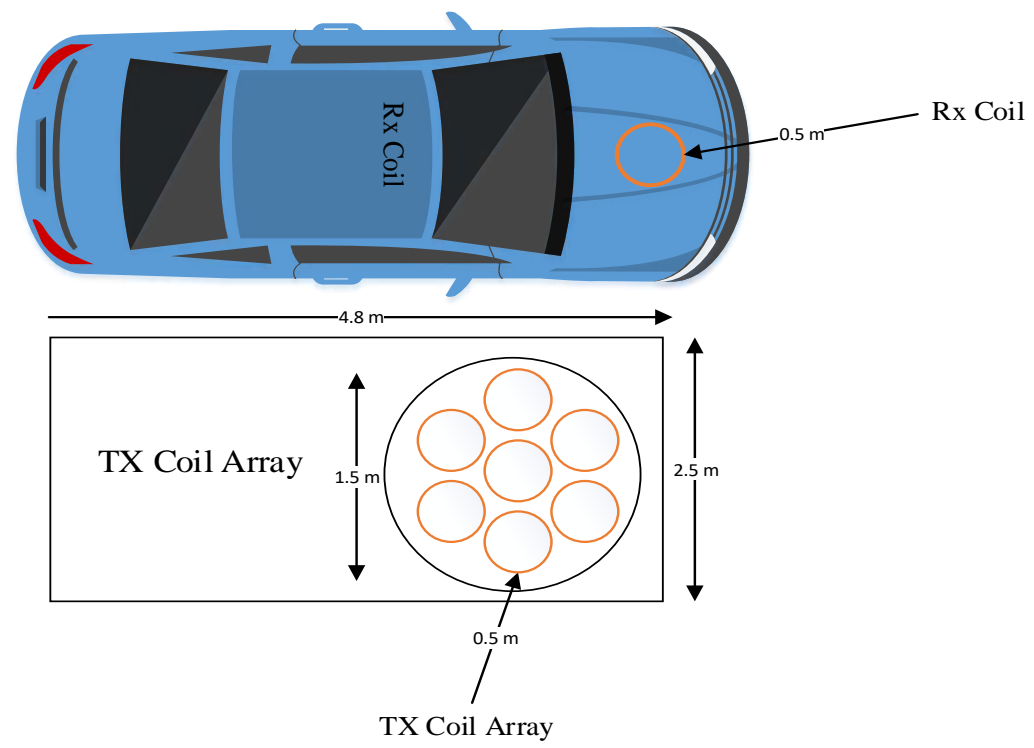

Fig. 2 - Illustration of 7 array circular coil [8]

We analyze and find that misalignment and weak coupling are primary problem in PTE transmission on EV between Tx and Rx. The latest study of circular coil array that has problem in the tangential boundary area will be our main guidance to conduct the research because of significant reduction of PTE. To do so, we will study PTE by using hexagonal coil arrays in a bigger misalignment position between $\mathrm{Tx}$ and Rx especially in the tangential boundary. This study has 
some sections. The next section will tell a short explanation of WPT and other related literature. The following section describes our design, data retrieval and experiment result. The last section provides analysis and conclusion.

\section{Wireless Power Transfer}

WPT is divided into radiation and non-radiation components [17]. WPT radiation is divided into Radio Frequency (RF) beam forming power directive and RF non-directive power transfers [18], [19]. Non-radiation WPT is classified into: magnetic inductive coupling, magnetic resonance coupling and capacitive coupling [9]. WPT Capacitive coupling uses the electric field of the capacitive coupling to the nearest transmission plane so that the area is limited [20]. This technology is rarely used because it requires high voltage to transfer significant power and the intensity of the electric field and it can harm the human body [10]. In WPT inductive magnetic coupling, the source moves the varying magnetic field that is connected to the primary coil and will induce the voltage across the receiving coil then transfer power to the existing load [11].

WPT magnetic resonance coupling uses the principle of resonance frequency to reduce leakage, making it possible to transfer power to longer distances where very strong electromagnetic fields are generated allowing to transfer large power. Transformers, electric machines, are examples of electrical system that use magnetic coupling, which are often regarded as contactless power transfer (CPT) or inductive power transfer (IPT) [9]. WPT radiation uses propagation of electromagnetic waves in distance with RF and microwaves as a medium for carrying energy that is differentiated into WPT RF beam forming power directive with limitations lies in the exact energy receiving location and non-directive RF WPT that works on the transmission of power distance far away by utilizing microwave radiation [21].

\subsection{Inductive Power Transfer}

The inductors that exchange magnetic flux with the others are called IPT [22]. Rx coil can be defined as the number of turns $\mathrm{N}_{2}$ in the destination whereas Tx coil is defined as the number of turns $\mathrm{N}_{2}$ from current source. Fig. 3 depicts the definition.

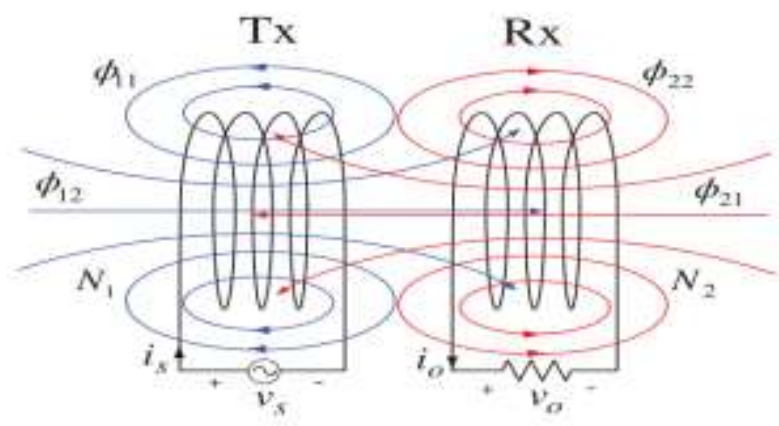

Fig. 3 - Flux magnet coupling coil [24]

IPT is governed by Ampere's circuit law and Faraday's inductive law [25]. Ampere's circuit law explains the changing intensity of the magnetic field around a closed loop L [24], which is equal to the electric current passing through the surface bounded by the loop, with the following equation:

$$
\nabla \times H=J=\oint_{L} H \cdot \mathrm{d} l=\int_{s} J \cdot \mathrm{ds}=I
$$

Where $\mathrm{A}$ is the current density [A / m2], I [A] is the current in the circuit and $\mathrm{H}$ [A / m] is the magnetic field [22]. Faraday's inductive law states that changes in magnetic fields per unit of time give rise to an electric motion force (emf). The general form of Faraday's law follows:

$$
\begin{gathered}
\nabla \times E=-\frac{\partial B}{\partial t} \Leftrightarrow \int_{L} E . d l=-\frac{d}{d t} \int_{S} B . d A=e m f \\
e m f=-\frac{d \phi}{d t}
\end{gathered}
$$


Mutual inductance between two circular loops separated by distance d with coil turns $n_{1}, n_{2}$, radius coil $r_{1}, r_{2}$ shown in Fig. 4 with permeability $\mu$ then it will be able to:

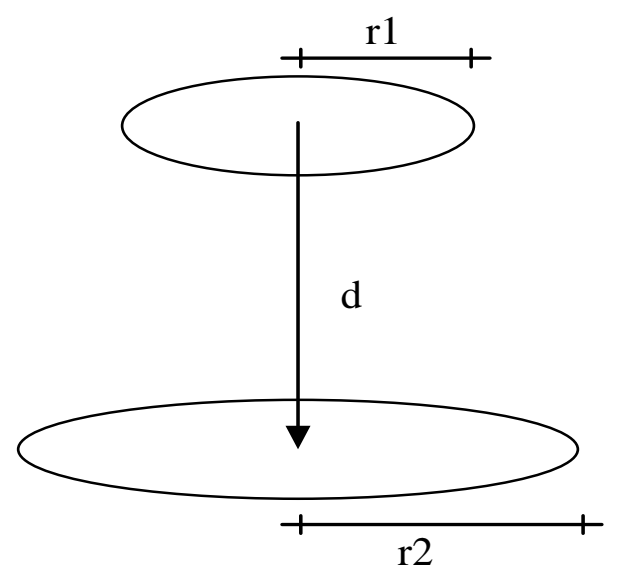

Fig. 4 - Two coil with distance $\mathbf{d}$ and radius $r_{1}$ and $r_{2}$

$$
M=\frac{\mu \pi n_{1} n_{2} r_{1}^{2} r_{2}^{2}}{\left[\left(r_{1}+r_{2}\right)^{2}+d^{2}\right] \sqrt{\left(r_{1}+r_{2}\right)^{2}+d^{2}}}
$$

And coupling Coefficient with equation:

$$
k=\frac{M}{\sqrt{L_{1} L_{2}}}
$$

IPT system needs high frequency for PTE. Meanwhile, it uses high frequency [26], the power factor (PF) is going to be smaller because of more inductive impedance [27]. Resistive impedance needs additional capacitive [28]. Additional capacitors makes the coils easily resonate in the circuit at the same frequency in order to create efficient PTE [29]. The oscillation with greater amplitude at some frequencies can be called resonance frequency of the system [30].

Equation 6 is the resonance frequency formula:

$$
f=\frac{1}{2 \pi \sqrt{L C}}
$$

The indication of the Quality Factor (Q-factor) can be used to detect energy loss rate. Equation 7 is known as Qfactor formula in RLC circuit:

$$
Q=\frac{1}{R \sqrt{\frac{L}{C}}}
$$

Equation 8 is formula of WPT efficiency:

$$
\eta \max \approx \frac{(k Q)^{2}}{\left(1+\sqrt{\left(1+(k Q)^{2}\right)}\right)^{2}}
$$

The coupling between the two coils is directly proportional to its mutual inductance and also designing coils that tolerate better misalignment. 


\subsection{Alignment in WPT}

While the Tx and Rx coils are aligned at a distance $d$, it means perfect alignment. The misalignment position determines PTE as an important factor as shown in fig. 5. There are 2 types of misalignment:

- Angular misalignment: when Rx coil position is at an angle $\theta$ even though it is parallel to the Tx coil and aligned well (Fig.5a).

- Lateral misalignment: when a pair of Tx and Rx coils in parallel position, the center has a horizontal distance $\Delta$ and vertical distance $\mathrm{d}$ when the Rx coil position is at an angle $\theta$ even though it is parallel to the Tx coil and aligned well (Fig.5b).

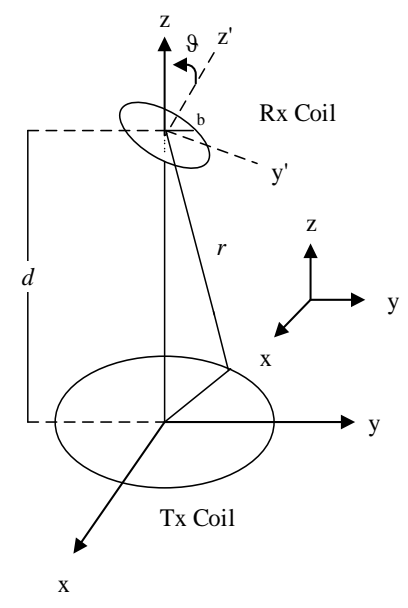

(a) Angular

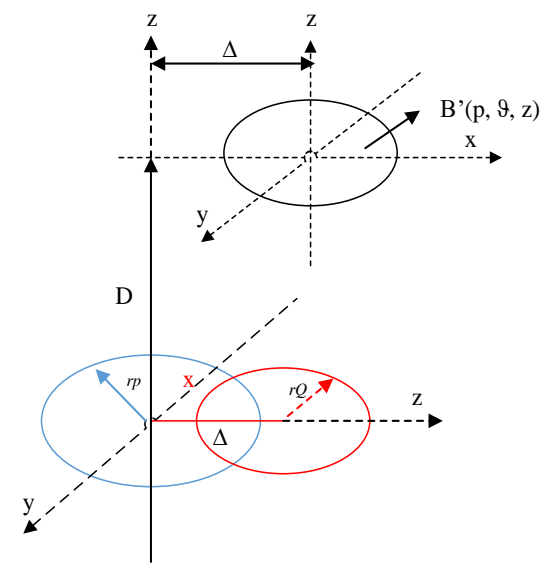

(b) Lateral

Fig. 5 - Misalignment [18]

\section{System Design of Receiver and Transmitter}

Fig. 6a displays our design of Tx coil consisting of a 555 timer in a stable mode, an LC primary resonance coil, a MOSFET, a rectifier and a DC power supply unit. Besides that, fig. 6b exhibits our design of Rx coil consisting of rectifier, secondary resonance coil and load in the form.

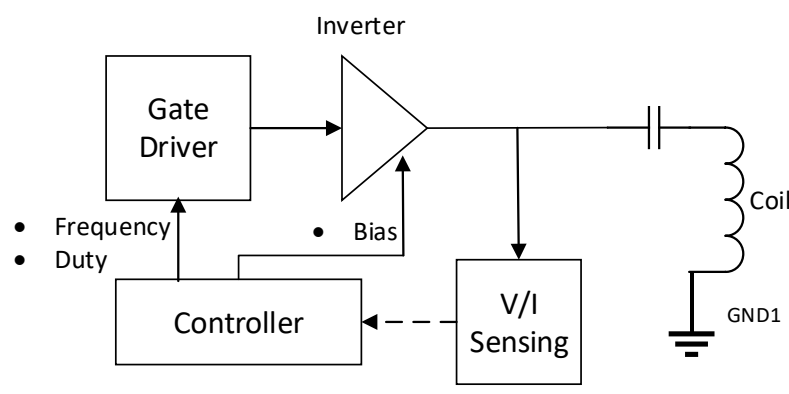

(a) Transmitter

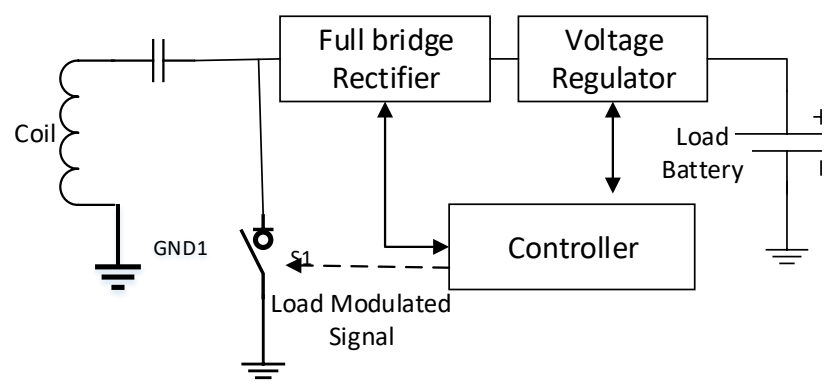

(b) Receiver

Fig. 6 - System design

The AC source flows with a main supply of $230 \mathrm{~V} \mathrm{AC} 50 \mathrm{~Hz}$ down to $12 \mathrm{~V}$ using a Step down $12 \mathrm{~V}$ Transformer, fed to the wave rectifier bridge which converts the AC signal to DC then as input to the LM7805 voltage regulator. The output of the 7805 regulator is supplied to Timer 555 which functions to produce a frequency of $22.2 \mathrm{kHz}$. The output from the timer is given to the MOSFET. The primary coil resonates at a frequency of around $22.2 \mathrm{kHz}$ to transfer the induced voltage in the air. When the secondary coil is placed close to the primary coil, because the electromotive force that induces the secondary coil is then fed to the high frequency rectifier to increase the high frequency AC. After that it 
is forwarded to the capacitor to filter out the noise that occurs in the AC and a DC voltage of $12 \mathrm{~V}$ is obtained. This DC is fed to the DC fan.

\subsection{Coil Design}

We design a new coil array to drive out the misalignment issue in tangential boundary as we defined. The new design will achieve higher PTE in tangential boundary using 7 array circle coil through comparison to the single coil benchmark [31]. We use previous study as a benchmark for measuring and retrieving data [16] and reconstruct the previous coil design (circle coil) to be our new design (hexagonal coil).

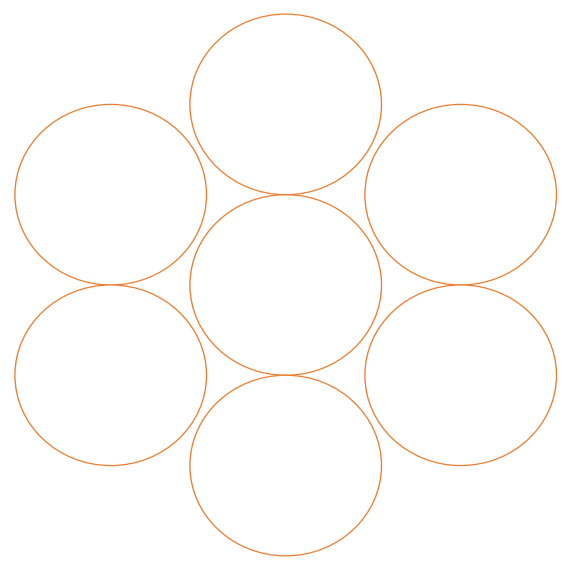

(a) 7 Array Circle Coil

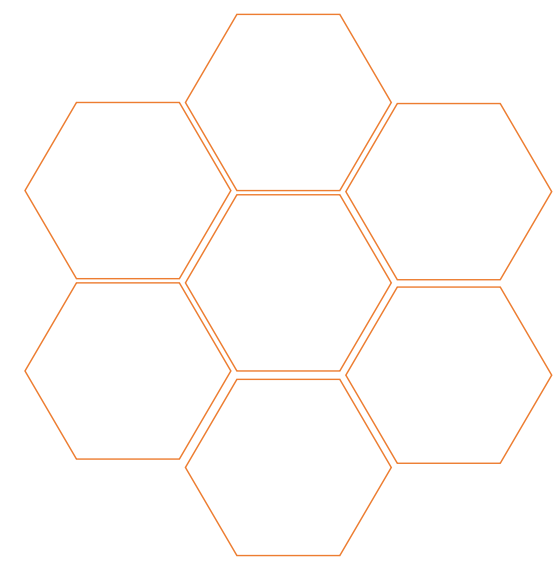

(b) 7 Array Hexagonal Coil

Fig. 7 - Coil design

2 coil designs are as follows:

- $\quad$ Reference coils (7 array circle coil) [8]: both primary (7 array circle coil) and secondary (1 circle coil) coils are $2.5 \mathrm{~cm}$ in diameter (Fig.7a).

- $\quad$ New coils ( 7 hexagonal coil array): both primary (7 hexagonal coil array) and secondary (1 circle coil) coils are $1.5 \mathrm{~cm}$ side size (Fig.7b).

As a benchmark, we use single loop coil which EV uses 1 coil on the Rx and 1 coil on the Tx.

\subsection{Measurement Method}

We use LCR meter to test and measure reactance, resistor and capacitor. Meanwhile, multi meter is used to measure voltage and the current. The oscilloscope is used to measure the resonant frequency.

We define our measurement in table 1. All the measurements are performed such as: (1) benchmark in X/Y axis (reference coil), and (2) performed arrays ( 7 circles arrays and 7 hexagonals arrays) on the central boundary and tangential boundary positions. The voltage and current on the side of the primary coil as well as the secondary coil are included in the measured parameters.

Table 1 - Parameter

\begin{tabular}{llll}
\hline Property & $\mathbf{7}$ array circle coil & $\mathbf{7}$ array hexagonal coil & Benchmark coil \\
\hline Air Gap & $10 \mathrm{~mm}$ & $10 \mathrm{~mm}$ & $10 \mathrm{~mm}$ \\
\hline $\mathrm{Rx}$ & $0.014 \mathrm{mH}, 0.9 \mathrm{Ohm}$ & $0.014 \mathrm{mH}, 0.9 \mathrm{Ohm}$ & $0.014 \mathrm{mH}, 0.6 \mathrm{Ohm}$ \\
\hline $\mathrm{Tx}$ & $0.014 \mathrm{mH}, 0.8 \mathrm{Ohm}$ & $0.014 \mathrm{mH}, 0.8 \mathrm{Ohm}$ & $0.014 \mathrm{mH}, 0.6 \mathrm{Ohm}$ \\
\hline Turns(N) & 30 & 28 & 9 \\
\hline Shaped & Circle & Hexagon & Circle \\
\hline Freq & $22.2 \mathrm{kHz}$ & $22.2 \mathrm{kHz}$ & $22.2 \mathrm{kHz}$ \\
\hline Coil D & $25 \mathrm{~mm}$ & (sides) $15 \mathrm{~mm}$ & $50 \mathrm{~mm}$ \\
\hline
\end{tabular}

The Air gap between the primary coils and secondary coils is $10 \mathrm{~mm}$. Fig. 8 shows the flowchart of measurement steps for each model of the coil. The measured parameters include the voltage and current on the side of the primary coil as well as the secondary coil side, the misalignment position at the central boundary position and tangential boundary shown in Figure 8. 


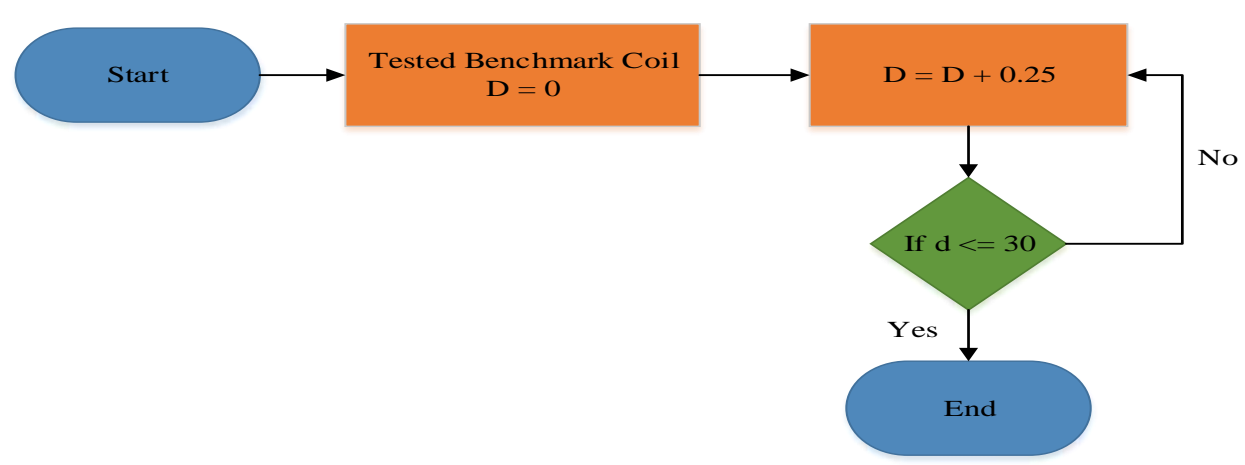

(a) Benchmark Coil

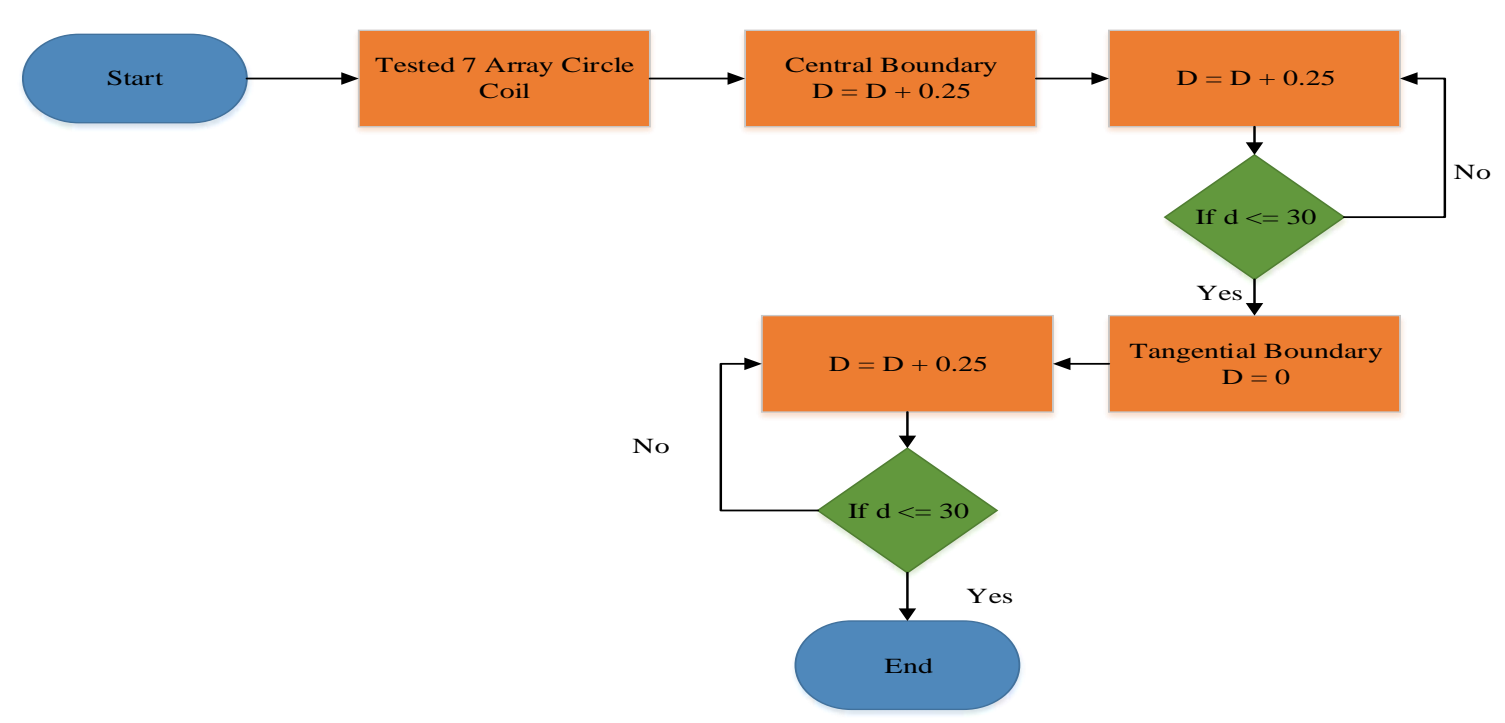

(b) 7 Array Circle Coil

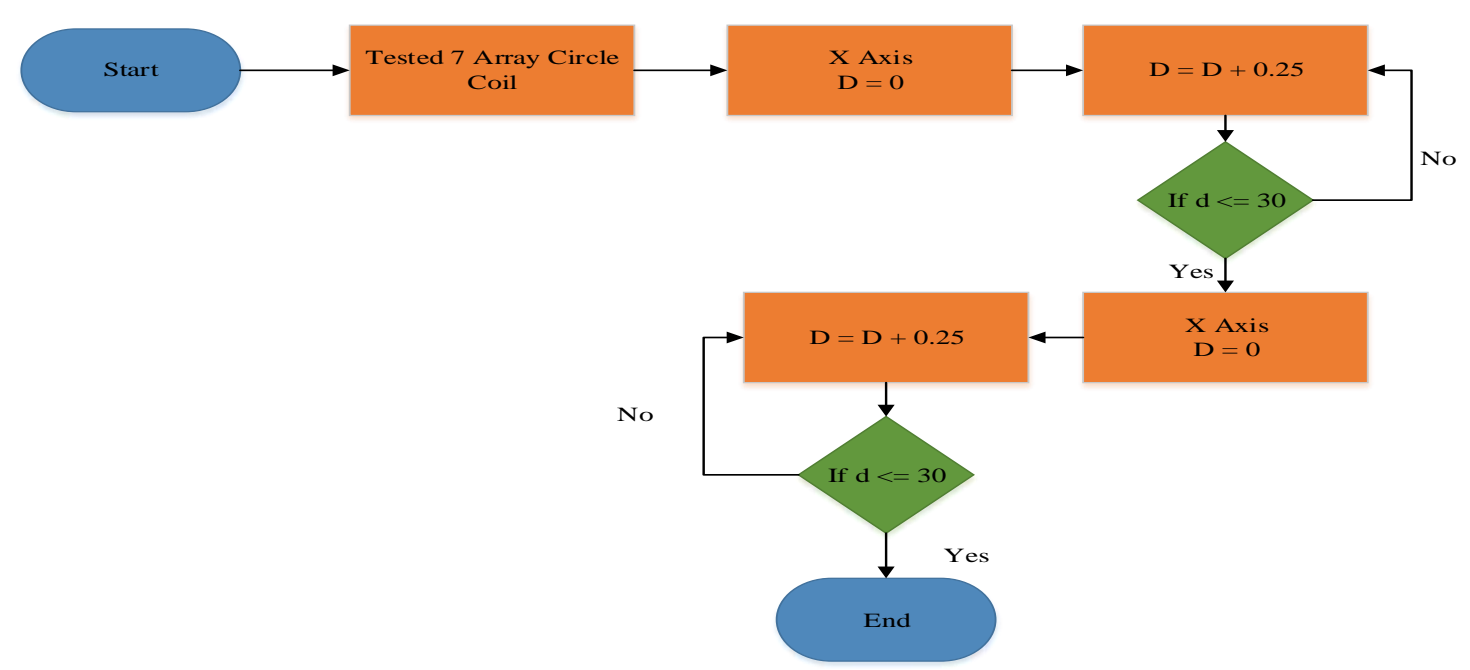

(c) 7 Array Hexagonal Coil

Fig. 8 - Flowchart measurement 


\section{Result and Discussion}

We carried out the experiments with some references to the model references. We reconstructed the experiment with our defined parameters in table 1.

For benchmark coils, the results obtained from the experiment with a trend approaching reference. With a circle coil with diameter of $50 \mathrm{~mm}$, the decrease in efficiency approaches curve in its misalignment distance when misaligned position is more than $10 \mathrm{~mm}$.

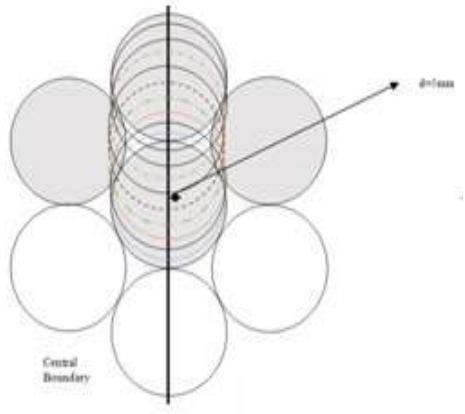

(a) Central boundary 7 array circle coil

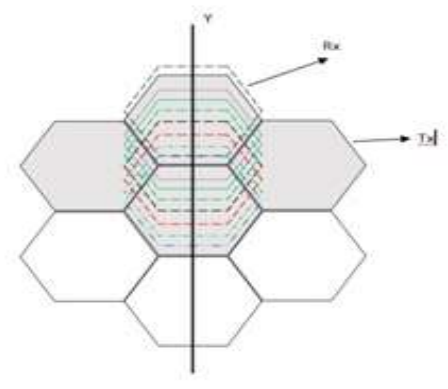

(c) Central boundary 7 array hexagonal coil

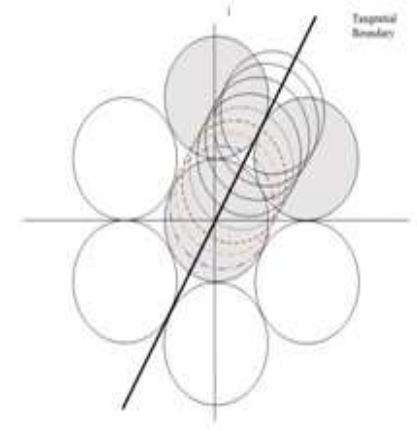

(b) Tangential boundary 7 array circle coil

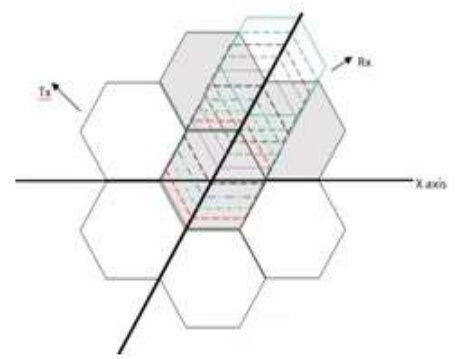

(d) Tangential boundary 7 array hexagonal coil

Fig. 9 - Measurement position

Our experiments on 7 circle coil arrays, we measured the central boundary and tangential boundary positions with circle diameters of $25 \mathrm{~mm}$. In the reconstruction of the central boundary measurement, the reconstruction results show that there is a sharp decline in the misalignment position of $5 \mathrm{~mm}$ to $12.5 \mathrm{~mm}$, where the lowest efficiency position can be in the $12.5 \mathrm{~mm}$ misalignment then it will rise up to its alignment position. The reconstruction of the tangential boundary measurements shows the same result up to $12.5 \mathrm{~mm}$ misalignment position with the central boundary. Based on heat map analysis in Fig.12b where in the tangential boundary position there is an empty gap when the position more than 12.5 $\mathrm{mm}$, efficiency decreased to $2 \%$ then rose slightly until the position of misalignment $22.5 \mathrm{~mm}$ then decrease to $0 \%$. The experienced increases when position of $17.5 \mathrm{~mm}$ to $22.5 \mathrm{~mm}$, because at this position, there is an influenced by the coil array next to it as described in Fig.11b. 


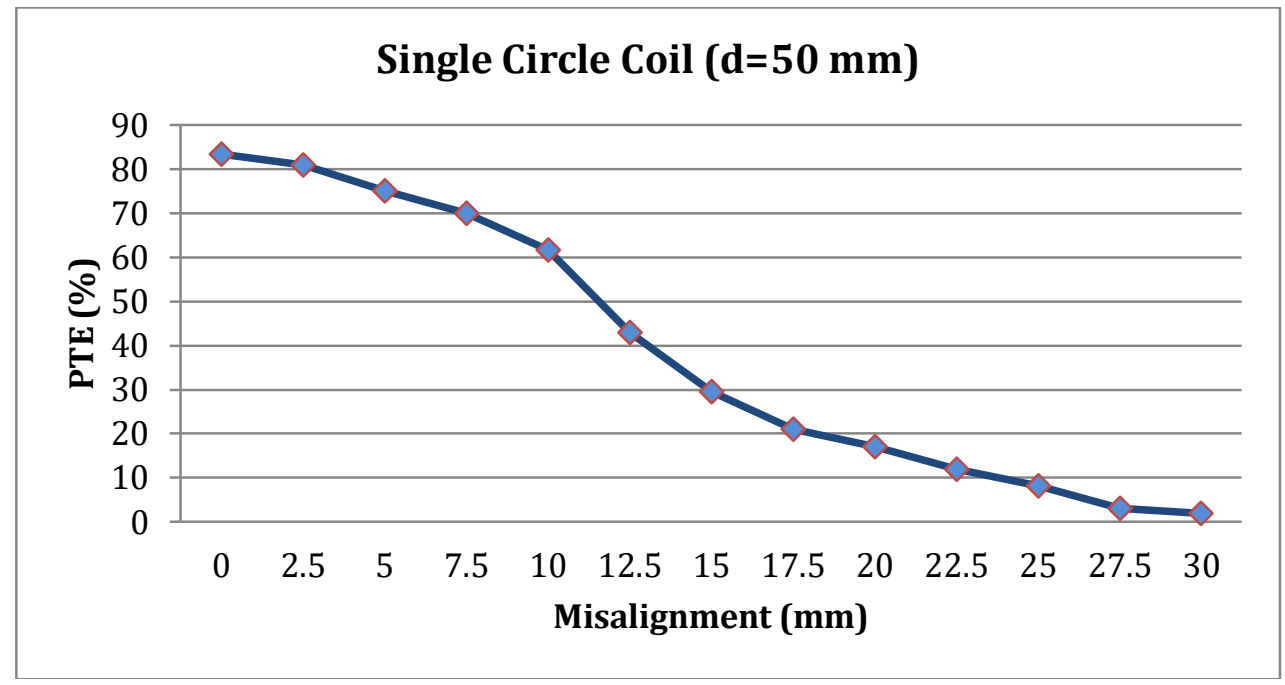

Fig. 10 - Measurement result benchmark coil

Measurement using the proposed coil design 7 hexagonal coil arrays, the position of the central boundary refers to the position of the Y axis in Fig. 10. The comparison between 7 array circle coils with hexagonal arrays is not significantly different.

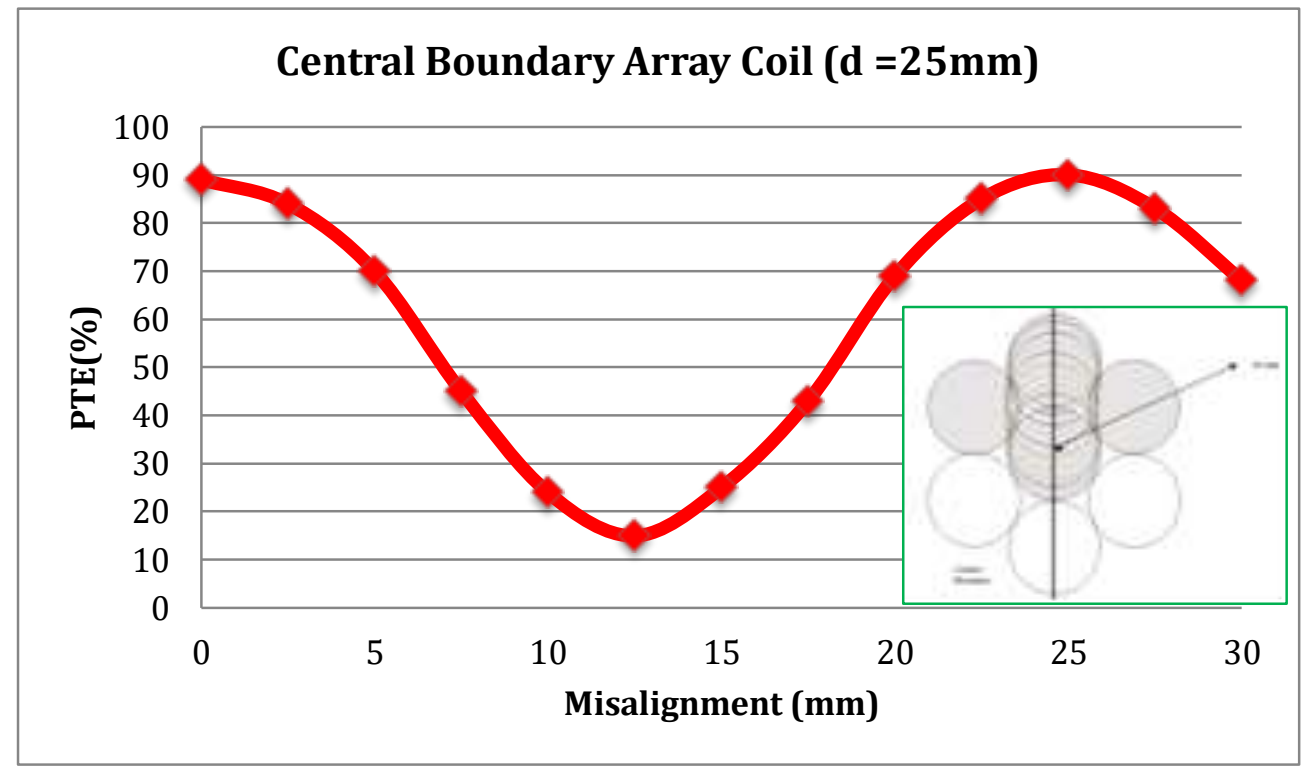

(a) Central boundary 


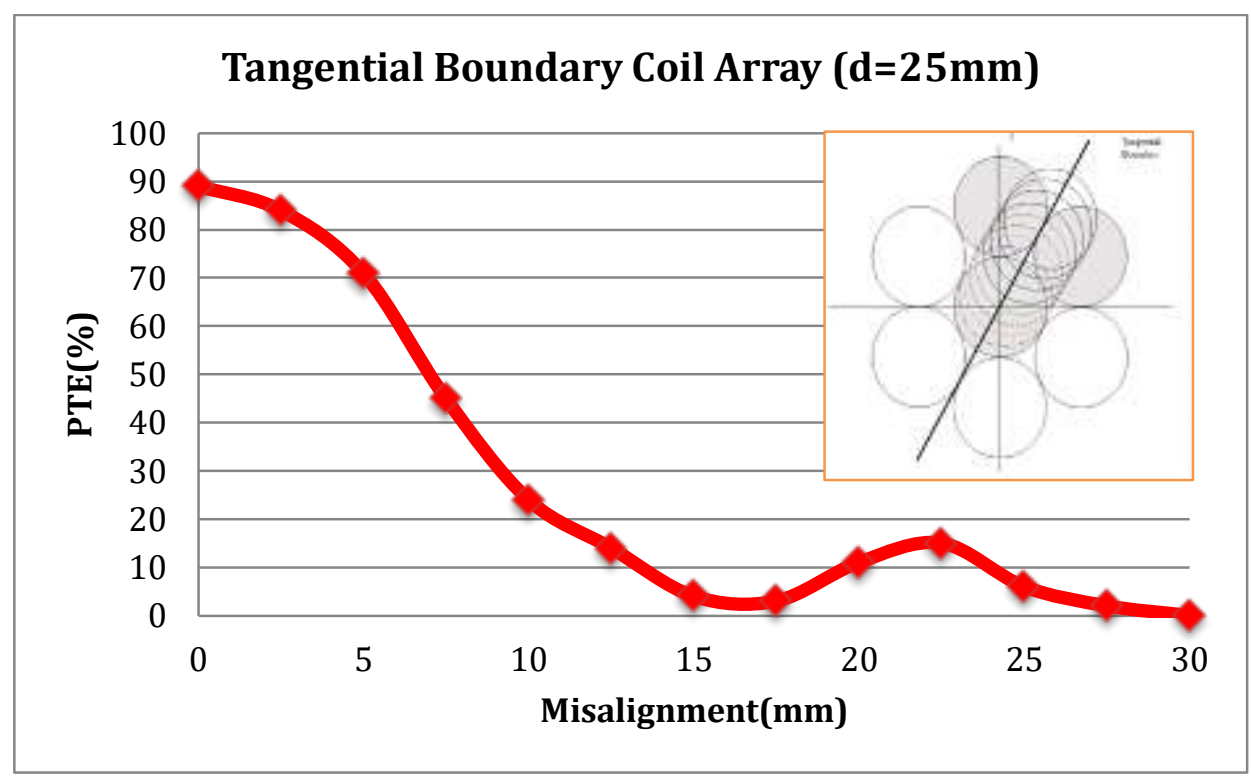

(b) Tangential Boundary

Fig. 11 - Measurement result of 7 array circle coil

Fig.12 displays heat map 7 array circular and 7 array hexagonal taken from a measurement matrix at 2.5 mm intervals for single-loop coils and hexagonal coil, then results in plots using 2D Graph on the MATLAB Heat Map. In the Fig.12b seen for 7 circle coil arrays, there is an empty gap when the position is more than $12.5 \mathrm{~mm}$ which means the effect of the coil is reduced.

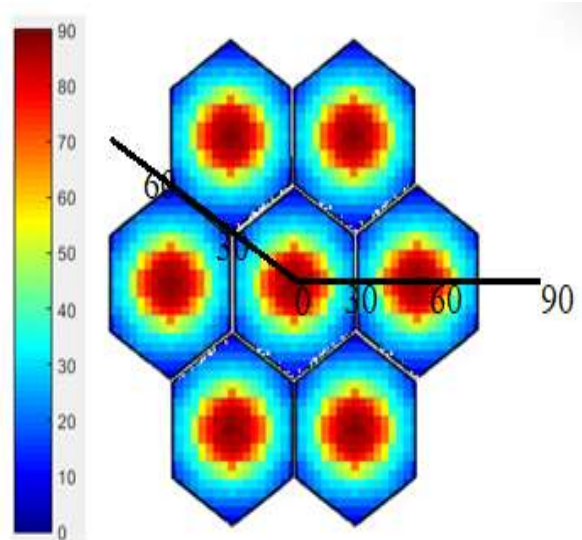

(a) 7 Array hexagonal coils
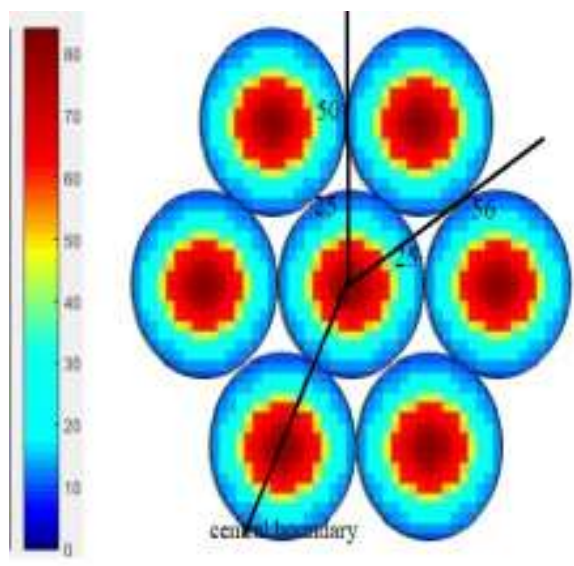

(b) 7 Array circle coils

Fig. 12 - Heat map relationship PTE and misalignment 


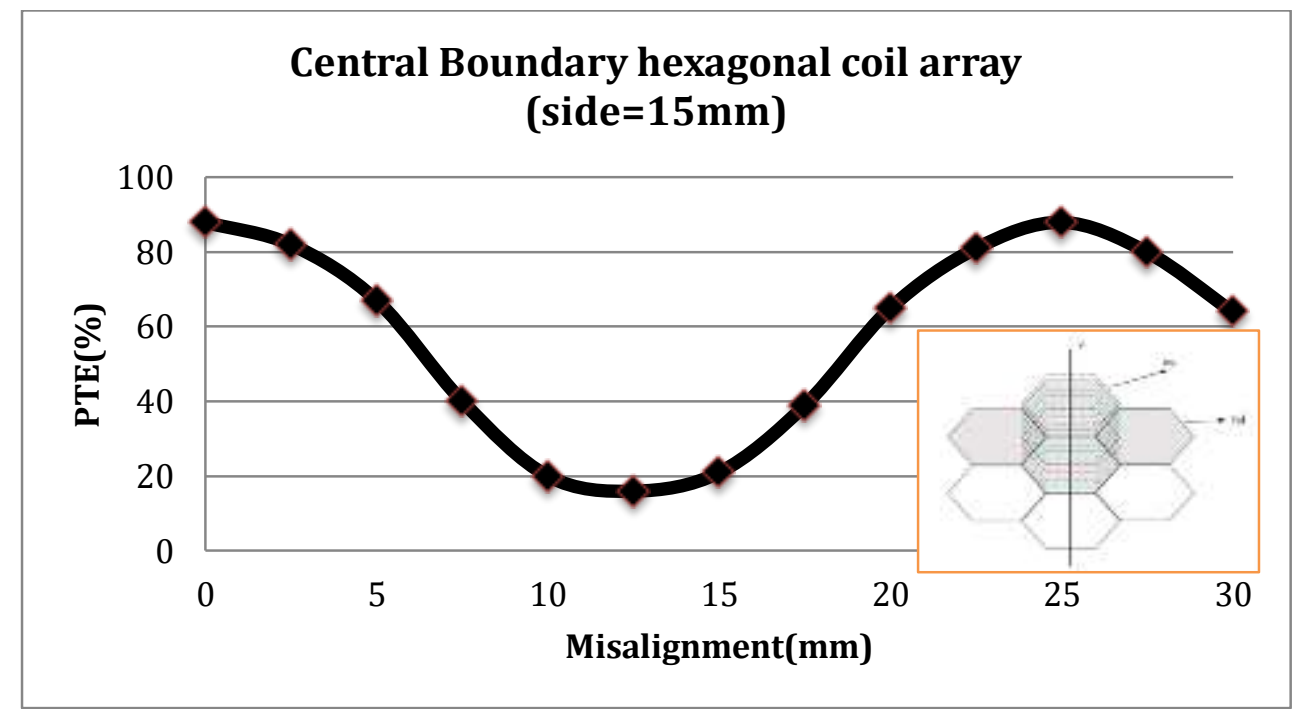

(a) $\mathrm{Y}$ axis

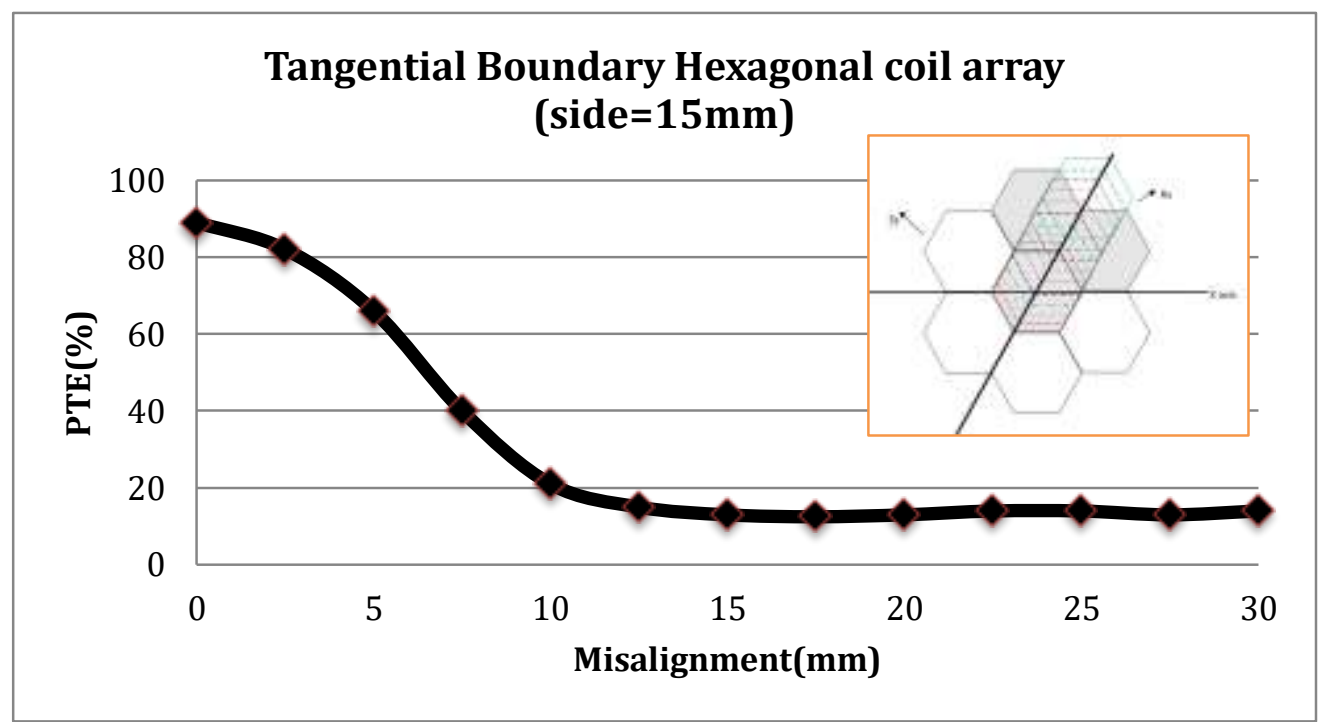

(b) X Axis/Tangential boundary

Fig. 13 - Measurement result of 7 array hexagonal coil

In tangential boundary position, there is no gap so that the efficiency level between the hexagonal and the circle does not the fundamental difference as shown in Fig. 14, Unlike the case with the measurement in the tangential boundary position as in Fig. 15. Measurement using 7 hexagonal coil arrays shows the efficiency at $15 \mathrm{~mm}$ misalignment position and stable to $30 \mathrm{~mm}$ misalignment position. Although the position of misalignment was stay away but the efficiency still at the same level. 


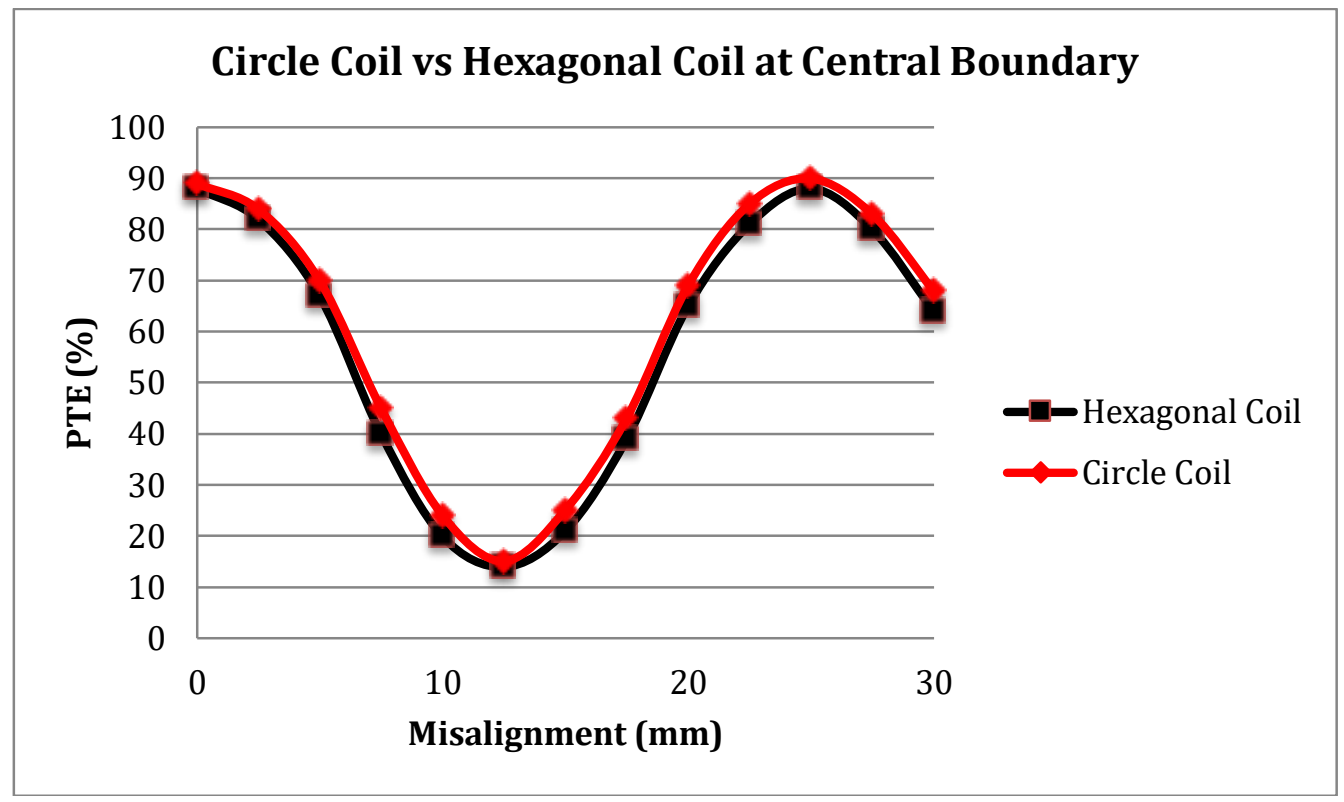

Fig. 14 - Comparison in central boundary

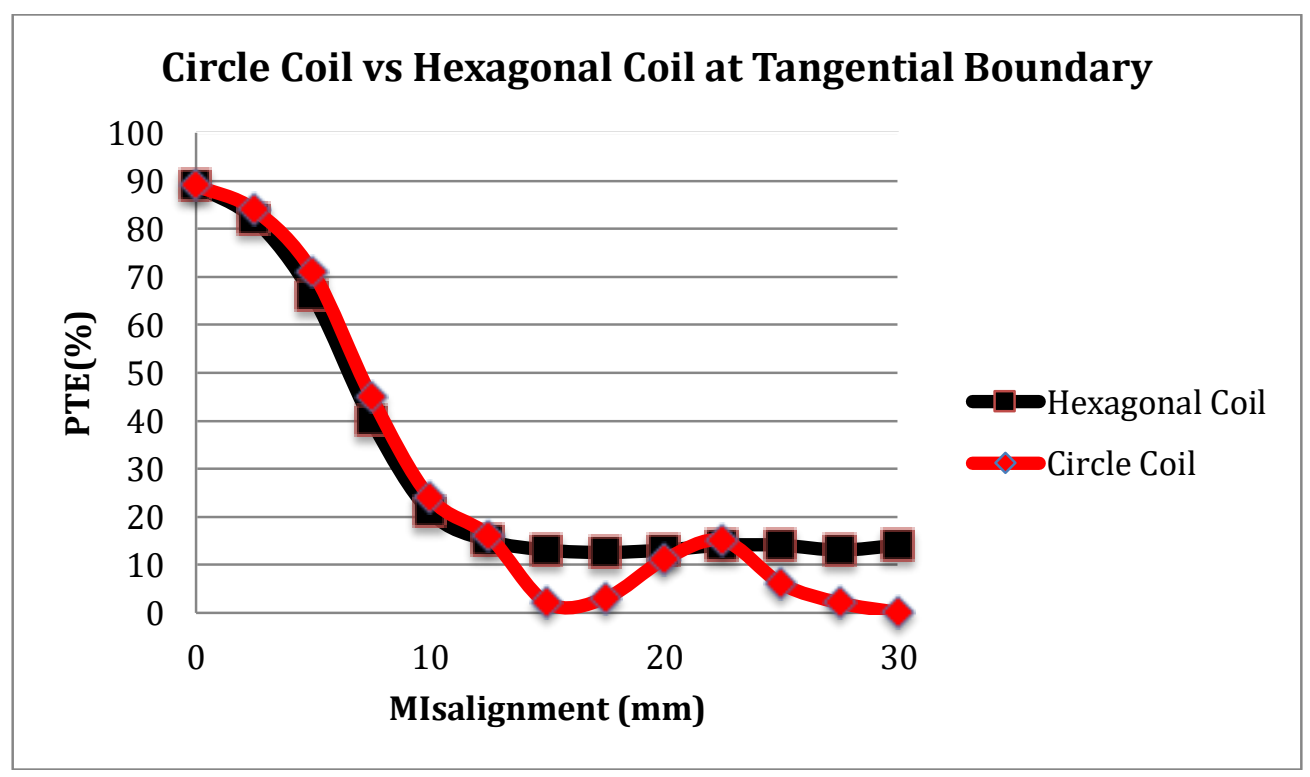

Fig. 15 - Comparison in tangential boundary

\section{Conclusion}

PTE is influenced by coil parameters that we have defined such as its dimensions, geometry and location. This study also solves misalignment problem in circle array types by introducing the main resonant coil design of hexagonal array types and then achieves much better PTE in boundary tangential position without losing PTE in central boundary position. If we see the driver's parking behavior, coil misalignment is unavoidable because of inaccurate car position. The results obtain an improvement of PTE observed at the tangential boundary position up to $12 \%$. In addition, the results also prove that the array design can reduce the radius of the Rx coil and then reduce the weight of the car.

PTE reduction between coils along tangential boundaries can be optimized by using hexagonal-shaped coils without reducing the maximum misalignment distance between coils. Our new coil design only resolves PTE associated with coil misalignment and ignores the losses caused by the additional circuits required in a complete EV charging system. 


\section{Future work}

It is necessary to design a system that results in increased power and higher hardware to work at the position of the Tx array. While it is simultaneously supplying energy to the Rx coil without reducing the need for input power.

\section{Acknowledgement}

We would like to thank Department of Computer Engineering, Universitas Wiralodra and Department of Electrical Engineering, Universitas Mercu Buana for research support.

\section{References}

[1] M.R. Basar, M.Y. Ahmad, J. Cho, F. Ibrahim (2016). An improved resonant wireless power transfer system with optimum coil configuration for capsule endoscopy, Sensors and Actuators A. Physical, 249, 207-216.

[2] D.H. Gawali, V.M. Wadhai (2017). Recent trends in energy management of wireless wearable bio sensor design. Proceedings of 2017 11th International Conference on Intelligent Systems and Control, ISCO 2017, 408-412.

[3] S. Das Barman, A.W. Reza, N. Kumar, M.E. Karim, A.B. Munir (2015). Wireless powering by magnetic resonant coupling: Recent trends in wireless power transfer system and its applications. Renewable and Sustainable Energy Reviews, 51 (2015) 1525-1552.

[4] S. Niu, H. Xu, Z. Sun, Z.Y. Shao, L. Jian (2019). The state-of-the-arts of wireless electric vehicle charging via magnetic resonance: principles, standards and core technologies. Renewable and Sustainable Energy Reviews, 114, 109302.

[5] S. Luo, S. Li, H. Zhao (2017). Reactive power comparison of four-coil, LCC and CLC compensation network for wireless power transfer. 2017 IEEE PELS Workshop on Emerging Technologies: Wireless Power Transfer, WoW 2017. 4, 268-271.

[6] R. Bhujade, R. Mujavar, P. Singh, B. Joshi, R. Oruganti (2016). Modeling and analysis of coupled coils for Wireless Power Transfer. 2016 IEEE International Conference on Power Electronics, Drives and Energy Systems (PEDES), IEEE, pp. 1-6.

[7] S. Seshadri, M. Kavitha, P.B. Bobba (2018). Effect of coil structures on performance of a four-coil WPT powered medical implantable devices. 2018 International Conference on Power, Instrumentation, Control and Computing (PICC), IEEE, pp. 1-6.

[8] X. Lu, P. Wang, D. Niyato, D.I. Kim, Z. Han (2016). Wireless Charging Technologies: Fundamentals, Standards, and Network Applications. IEEE Communications Surveys \& Tutorials. 18, 1413-1452.

[9] M. Debbou, F (2016). Colet, Inductive wireless power transfer for electric vehicle dynamic charging. 2016 IEEE PELS Workshop on Emerging Technologies: Wireless Power Transfer (WoW), IEEE, pp. 118-122.

[10] S. Chatterjee, A. Iyer, C. Bharatiraja, I. Vaghasia, V. Rajesh (2017). Design Optimisation for an Efficient Wireless Power Transfer System for Electric Vehicles. Energy Procedia. 117, 1015-1023.

[11] S.A. Birrell, D. Wilson, C.P. Yang, G. Dhadyalla, P. Jennings (2015). How driver behaviour and parking alignment affects inductive charging systems for electric vehicles. Transportation Research Part C: Emerging Technologies, $58,721-731$.

[12] W. Zhang, J.C. White, A.M. Abraham, C.C. Mi (2015). Loosely Coupled Transformer Structure and Interoperability Study for EV Wireless Charging Systems. IEEE Transactions on Power Electronics, 30, 6356-6367.

[13] H. Kim, C. Song, D.-H. Kim, D.H. Jung, I.-M. Kim, Y.-I. Kim, J. Kim, S. Ahn, J. Kim (2016). Coil Design and Measurements of Automotive Magnetic Resonant Wireless Charging System for High-Efficiency and Low Magnetic Field Leakage. IEEE Transactions on Microwave Theory and Techniques, 64, 1-18.

[14] F. Turki, V. Staudt, A. Steimel (2015). Dynamic wireless EV charging fed from railway grid: Grid connection concept. 2015 International Conference on Electrical Systems for Aircraft, Railway, Ship Propulsion and Road Vehicles (ESARS), IEEE, pp. 1-5.

[15] Z. Dang, J.A.A. Qahouq (2014). Modeling and Investigation of Magnetic Resonance Coupled Wireless Power Transfer System with Lateral Misalignment. Applied Power Electronics Conference and Exposition (APEC), 2014 Twenty-Ninth Annual IEEE, pp. 1317-1322.

[16] X. Mou, O. Groling, H. Sun (2017). Energy-Efficient and Adaptive Design for Wireless Power Transfer in Electric Vehicles. IEEE Transactions on Industrial Electronics, 64, 7250-7260.

[17] A. Ahmad, M.S. Alam, Y. Rafat, S. Shariff (2020). Designing and demonstration of misalignment reduction for wireless charging of autonomous electric vehicle. eTransportation, 4, 100052.

[18] X. Mou, O. Groling, H. Sun (2017). Energy-Efficient and Adaptive Design for Wireless Power Transfer in Electric Vehicles. IEEE Transactions on Industrial Electronics, 64, 7250-7260.

[19] B. Zhang, R.B. Carlson, J.G. Smart, E.J. Dufek, B. Liaw (2019). Challenges of future high power wireless power transfer for light-duty electric vehicles technology and risk management. eTransportation, 2, 100012.

[20] X. Yang, C. Song, C. Zhu, C. Liang, R. Sun (2019). Impacts of Misalignments on Mesh Behaviors of Face-Hobbed Hypoid Gear Considering System Deformation. IEEE Access, 7, 79244-79253. 
[21] Y. Li, J. Zhao, Q. Yang, L. Liu, J. Ma, X. Zhang (2019). A Novel Coil with High Misalignment Tolerance for Wireless Power Transfer. IEEE Transactions on Magnetics, 55, 1-4.

[22] J. Xu, Y. Xu, Q. Zhang (2019). Calculation and analysis of optimal design for wireless power transfer. Computers \& Electrical Engineering, 80, 106470.

[23] R. Shadid, S. Noghanian (2018). Study of Misalignment Effects on Hybrid Power Transfer and Wireless Antenna System for Implanted Devices. 2018 IEEE International Symposium on Antennas and Propagation \& USNC/URSI National Radio Science Meeting, IEEE, pp. 2553-2554.

[24] X. Liu, W. Han, C. Liu, P.W.T. Pong (2018). Marker-Free Coil-Misalignment Detection Approach Using TMR Sensor Array for Dynamic Wireless Charging of Electric Vehicles. IEEE Transactions on Magnetics, 54, 1-5.

[25] Pingan Tan, Saiqi Cao, Xieping Gao (2016). Adjustable coupler for inductive contactless power transfer system to improve lateral misalignment tolerance. 2016 IEEE 8th International Power Electronics and Motion Control Conference (IPEMC-ECCE Asia), IEEE, pp. 2423-2426.

[26] B.J. Varghese, P.B. Bobba, M. Kavitha (2016). Effects of coil misalignment in a four coil implantable wireless power transfer system. 2016 IEEE 7th Power India International Conference (PICON), IEEE, pp. 1-6.

[27] D. Ustun, S. Balci, K. Sabanci (2020). A parametric simulation of the wireless power transfer with inductive coupling for electric vehicles, and modelling with artificial bee colony algorithm. Measurement, 150, 107082.

[28] S. Kuzey, S. Balci, N. Altin (2017). Design and analysis of a wireless power transfer system with alignment errors for electrical vehicle applications. International Journal of Hydrogen Energy. 42, 17928-17939.

[29] A. Abdolkhani (2016). Fundamentals of Inductively Coupled Wireless Power Transfer Systems. Wireless Power Transfer - Fundamentals and Technologies.

[30] A.A. Eteng, S.K.A. Rahim, C.Y. Leow, S. Jayaprakasam, B.W. Chew (2017). Low-power near-field magnetic wireless energy transfer links: A review of architectures and design approaches. Renewable and Sustainable Energy Reviews, 77 , 486-505.

[31] S.I.P. and V.O.M. Aderemi A. Atayero, Oluwaseun Ajijola (2016). Development of a wireless power transfer system using resonant inductive coupling, Lecture Notes in Engineering and Computer Science. 2225, $13-18$. 